\title{
La dimensión de independencia y dependencia de campo en educación: una revisión bibliométrica (2003-2013)
}

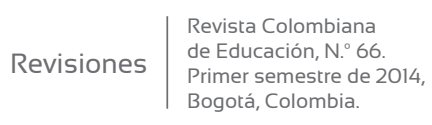

//Field-dependence and field-
independence in education: a
bibliometric review (2003-2013)

//A dimensão dependência-independência

de campo na educação: uma revisão

bibliométrica (2003-2013)

Aldo Hernández Barrios*
Recibido: 22/12/2013 Evaluado: 08/03/2014

\begin{abstract}
Magíster en Psicología con énfasis en Análisis del Comportamiento. Director del Centro de Investigaciones de Psicolo-
\end{abstract} gía. Bogotá, Colombia. Correo electrónico: aldo.hernandez@konradlorenz.edu.co

\section{Resumen}

El propósito de este estudio fue realizar una revisión bibliométrica de los artículos científicos publicados entre 2003 y 2013 en idioma inglés, asociados con el estilo cognitivo en su dimensión de independencia dependencia de campo (DIC) en el entorno educativo. Las fuentes de información fueron las bases de datos Science Direct ${ }^{\circ}$ y ERIC $($, un total de 59 artículos fueron depurados en una base de datos y se procedió a realizar análisis descriptivos. Entre otros aspectos comunes derivados de la revisión, se encuentra una prevalencia de los estudios correlacionales, el uso del GEFT como instrumento preferido para evaluar la DIC, la participación de estudiantes universitarios como muestra y unos títulos de revistas con mayor frecuencia de publicaciones asociadas al tema. Se plantean algunos interrogantes respecto al desarrollo del área en la última década.

\section{Abstract}

The purpose of this study was to undertake a bibliometric review of scientific papers written in English language and published between 2003 and 2013. The papers are associated with the cognitive style in its field-dependence (FD) and field-independence (FI) dimension in the educational context. The sources of information were Science Direct ${ }^{\circledR}$ and ERIC $®$ databases. Fifty-nine papers were filtered in a database and then a descriptive analysis was undertaken. Among the common aspects derived from the review, it is noticeable the prevalence of correlational studies, the use of GEFT as preferred instrument to evaluate the $\mathrm{FD}$ and $\mathrm{FI}$, the participation of university students as samples and some journals titles with high frequency of publication associated to this subject. FinaIly, we posed some questions regarding the development of this area in the last decade.

\section{Resumo}

O objetivo deste estudo foi realizar uma revisão bibliométrica dos artigos científicos publicados em inglês entre 2003 e 2013, associados ao estilo cognitivo na dimensão dependência-independência de campo no meio educacional. As fontes de informação foram as bases de dados Science Direct ${ }^{\circledR}$ e ERIC®. 59 artigos foram incluídos num banco de dados para realizar análises descritivas. Achou-se prevalência de estudos correlacionais, uso do GEFT como principal

\section{Palabras Clave}

Estilo cognitivo, dependencia de campo, independencia de campo, bibliometría, educación.

\section{Keywords}

Cognitive style, field-dependence, field-independence, bibliometry, education.

\section{Palavras chave}

Estilo cognitivo, dependênciaindependência de campo, bibliometria, educação. 
instrumento para avaliar a dependência-independência de campo, a seleção de estudantes universitários nas amostras de estudo, e algumas revistas com mais frequência de publicações associadas ao tema. Formulam-se questões respeito do desenvolvimento da área na última década.

\section{Introducción}

Un fenómeno perceptual identificado por Witkin (1950, 1964, 1965, 1976; Witkin y Ash, 1948) consistente en la presencia de diferencias individuales frente a la tarea de ubicar en posición vertical de una barra inscrita dentro de un marco inclinado (Test del marco y la varilla; RFT) fue descrito y conceptualizado como una dimensión de estilo cognitivo. Se entiende por estilo cognitivo un modo relativamente estable, integrador y diferenciador en la percepción y procesamiento de la información (Hederich, 2010). Witkin clasificó a los participantes de sus estudios en independientes de campo (IC; aquellos que establecen la verticalidad de manera precisa gracias a que atienden a la información propioceptiva vestibular) y en dependientes de campo (DC; aquellos que fallan al establecer la verticalidad porque toman como base referentes visuales externos, en este caso el marco). La dependencia independencia de campo (DIC) representa un estilo cognitivo en tanto se relaciona con diferentes funciones cognitivas y conductuales, haciendo énfasis en que los IC usan claves internas para evaluar la verticalidad, poseen altas habilidades para el desenmascaramiento perceptual, altos niveles de lateralización hemisférica, mientras que los individuos DC presentan un funcionamiento global, tendencia a la interacción social y a mantener la estructura de las representaciones en su forma original.

La DIC ha sido evaluada principalmente con el RFT (tarea que consiste en calificar la verticalidad de una barra en relación a un marco cuadrado con diferentes inclinaciones) y con el test de figuras enmascaradas (EFT; que consiste en identificar una figura simple dentro de una figura compleja). No obstante, algunos investigadores han propuesto que la DIC representa únicamente una habilidad perceptual (Zhang, 2004) y que el RFT y el EFT no son instrumentos precisos ya que carecen de control espacial de la elevación (Hudson, Li y Matin, 2006).

Los estudios tienden a mostrar que hay relación entre las ejecuciones en el EFT y el desempeño académico de los estudiantes (Hederich y Camargo, 2000; Tinajero y Fernández, 1998; López, 
Hederich y Camargo, 2011) favoreciendo a los IC. De igual manera, hay una relación favorable entre profesores y alumnos con igual estilo cognitivo (Chao, Huang y Li, 2003; Tinajero, Castelo, Guisande y Páramo, 2011; Saracho, 1980).

En general, el área de estudio de la DIC ha estado caracterizada por trabajos empíricos; experimentales y correlacionales, que resultan críticos para la construcción del conocimiento científico. En procura de rastrear el estado actual de la investigación en DIC, el presente estudio está orientado al desarrollo de una revisión del tema, que comprende los desarrollos en la última década en las bases de datos más importantes de referencia científica de habla inglesa. Este trabajo puede complementar el panorama actual por Hederich (2013) cuyo énfasis se dirigió hacia las publicaciones iberoamericanas.

\section{Metodología}

Los métodos aplicados en este estudio están basados en los lineamientos establecidos por Petticrew y Roberts (2008), para revisiones en ciencias sociales.

\section{Búsqueda}

La búsqueda de la información se realizó entre septiembre y noviembre de 2013 en las bases de datos Science Direct ${ }^{\circledR}$ de la casa editorial Elsevier $^{\circledR}$ y ERIC $^{\circledR}$, una biblioteca on-line patrocinada por el Institute of Educational Service del Departamento de Educación de los Estados Unidos; la primera se escogió dado que contiene la información académica de mayor impacto científico, mientras que la segunda es el mayor repositorio de información en educación de Estados Unidos. La información de $\mathrm{ERIC}^{\circledR}$ fue provista a través del motor de búsqueda Academic Search Complete ${ }^{\circledR}$. Se usaron en los campos de búsqueda las siguientes expresiones: field independence, field independent, field dependence, field dependent. Estas se establecieron en los campos de: título, abstract y palabras clave a través del operador lógico "OR". Se limitó la búsqueda considerando la fecha de publicación, la cual debía ser mayor o igual a 2003; incluyendo aquellos aceptados para publicación (en prensa). Se filtró la búsqueda por artículos arbitrados y publicados en revistas científicas.

Se excluyeron los artículos cuya población presentaba trastornos mentales consignados en el DSMIV-TR de la American Psychiatric Association (Flores, 2012), adicionalmente aquellos que estaban circunscritos a otro campo diferente al de educación. El reporte de datos bibliográficos fue importado a un libro de Microsoft Excel ${ }^{\circledR} 2011$ para $\mathrm{Mac}^{\circledast}$. La base de datos fue depurada eliminando entradas de artículos mencionados dos o más veces prefiriendo la información obtenida en Science Direct ${ }^{\circledR}$, dado que en esta base de información se obtie- 
nen con mayor frecuencia los textos completos. Una vez depurada la base de datos se procedió a obtener los artículos en medio electrónico.

La base de datos resultante consideró los campos de: número de identificación de artículo, base de datos, tema (en el caso particular de este reporte, el tema es constante; field independencedependence), título de la revista, autores, título del artículo, resumen, referencia de acuerdo con el Publication Manual of the American Psychological Association (APA, 2009), número de citas reportadas en Google Scholar ${ }^{\circledR}$, las cinco primeras palabras claves reportadas en cada artículo, el país en el que se aplicó el procedimiento, la identificación de los participantes por grupo etario y por género; así como el $n$ muestral, hasta tres variables sometidas a análisis, hasta tres tipos de instrumentos usados para la medición de las variables, el tipo de estudio, el diseño y las conclusiones.

\section{Análisis de la información}

La base de datos obtenida se normalizó manualmente en cuanto a campos y caracteres y se importó a un visor de datos de IBM ${ }^{\circledR}$ SPSS $^{\circledast}$ Statistics versión 20 para Mac OS ${ }^{\circledast}$. Se procedió a realizar análisis descriptivos de las variables de la base de datos y tablas cruzadas para visibilizar las interacciones entre las variables de análisis. Se realizaron agrupaciones en los casos en que los valores reales tuvieron un alto grado de dispersión o poca frecuencia.

\section{Resultados}

La búsqueda en las bases de datos arrojó 104 artículos, de los cuales cuatro se identificaron como entradas duplicadas. Al aplicar los criterios de exclusión (que no pudieron ser implicados en la búsqueda experta en las bases de datos) se obtuvo un total de 59 artículos, de los cuales 44 fueron obtenidos de la base ERIC ${ }^{\circledR}$ y los 15 restantes de Science Direct ${ }^{\circledR}$. Se acudió a la base de datos APA PsyNET ${ }^{\circledR}$ para obtener aquellos artículos que no fueron obtenidos a texto completo de las bases de datos objetivo del estudio. También fue necesario usar Google Scholar ${ }^{\circledR}$ para identificar el número de citas (se optó por este motor de vista dado que muchas revistas de ERIC ${ }^{\circledR}$ no están indexadas por Scopus ${ }^{\circledR}$ ). Posterior al análisis inicial se decidió hacer uso de la información de $\mathrm{SCl}$ mago ${ }^{\circledR}$ (disponible en http://www.scimagojr.com) para identificar el cuartil de ubicación de algunas publicaciones a partir del SJR. 


\section{Producción anual y caracterización de los participantes}

En la figura 1 se presenta el número de artículos relacionados con el año de publicación. El año 2012 fue el periodo con más publicaciones en el área con un total de 12 artículos, seguido por el año 2010 (9 artículos) y con igual número los años 2004 y 2011. En contraste, en los años 2003 y 2006, en que solamente hubo dos publicaciones en cada uno.

Figura 1. Número de artículos publicados en el área por año entre 2003 y 2013.

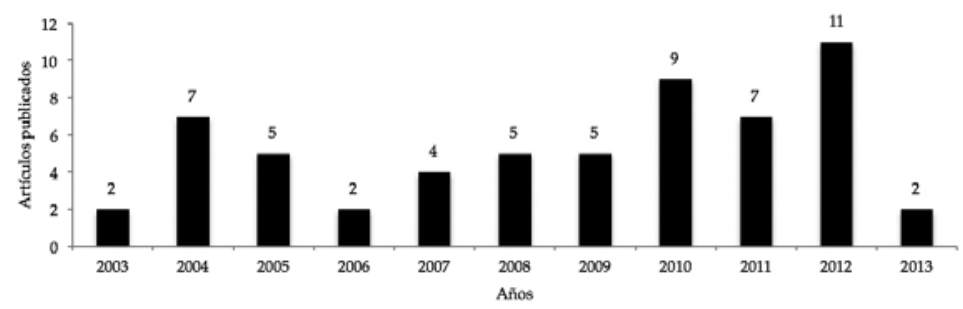

Los estudios tuvieron la participación de 7568 individuos. Pero no todos los estudios caracterizaron a sus participantes en términos de género y grupo etario. Solamente a 2446 participantes se les identificó tales características; 216 eran niños (107 hombres y 109 mujeres), 1095 adolescentes (430 hombres y 665 mujeres) y 1135 adultos (494 hombres y 641 mujeres).

\section{Aspectos bibliográficos}

Los artículos sobre DIC ubicados en esta búsqueda sistemática se han publicado en 42 revistas científicas arbitradas. En la tabla 1 se presenta el listado del nombre de las publicaciones y la cantidad de artículos publicados en el periodo de observación. De estos 24 títulos de revistas, 12 han publicado entre 2 y 4 artículos en el área de interés entre 2 y 4 veces. Cabe anotar que todos los estudios reportados en la tabla 1 son de carácter empírico correlacional.

Tabla 1. Revistas incluidas en ERIC ${ }^{\circledR}$ o Science Direct ${ }^{\circledR}$ que han publicado sobre DIC.

Revista

Computers \& Education

Educational Technology Research and Development

Personality and Individual Differences

Research in Science \& Technological Education

Turkish Online Journal of Educational Technology-TOJET

Computers in Human Behavior

Publicaciones DIC

4

4

3

3

3

2 


\section{Revista}

Electronic Journal of Research in Educational Psychology

International Journal of Information Management

International Journal of Instructional Media

International Journal of Science and Mathematics Education

Journal of Computer Assisted Learning

Procedia-Social and Behavioral Sciences

American Journal of Play

Asia Pacific Education Review

Calico Journal

E-Journal of Instructional Science and Technology

Early Child Development and Care

Educational Psychology

Educational Technology \& Society

European Review of Applied Psychology

Hacettepe University Journal of Education

Interactive Learning Environments

International Journal of Human-Computer Studies

International Journal of Science Education

Journal of Agricultural Education

Journal of American Indian Education

Journal of College Teaching \& Learning

Journal of Educational Computing Research

Journal of Educational Technology and Society

Journal of Pan-Pacific Association of Applied Linguistics

Journal of Science Teacher Education

Journal of Teaching in Physical Education

Journal of Technology Studies

Literacy Research and Instruction

Multicultural Education

Multicultural Perspectives

Physical Education and Sport Pedagogy

Science Education

Serialists

TESL Canada Journal
Publicaciones DIC

\begin{tabular}{|l|}
\hline 2 \\
\hline 2 \\
\hline 2 \\
\hline 2 \\
\hline 2 \\
\hline 2 \\
\hline 1 \\
\hline 1 \\
\hline 1 \\
\hline 1 \\
\hline 1 \\
\hline 1 \\
\hline 1 \\
\hline 1 \\
\hline 1 \\
\hline 1 \\
\hline 1 \\
\hline 1
\end{tabular}


El número de citas que ha recibido cada artículo fue obtenido a través de Google Scholar®, el rango de citación varía de 1 a 60. Nueve artículos (correspondientes al 15\%) han tenido 10 o más citaciones (tabla 2). El artículo de Zhang (2004) fue el más citado con 60 veces en el periodo 20042013.

Se inspeccionó el factor de impacto de las revistas en las que se publicaron los artículos más citados; el factor de impacto es un indicador asociado a la representatividad de la revista en la comunidad del conocimiento. La revista Computer and Education ha publicado tres de los artículos más citados; esta revista está en el cuartil superior (Q1) del Scimago Journal \& Country Rank (SJR) en el área de Educación. La publicación Personality and Individual Differences está ubicada en el Q1 SJR en las áreas de medicina y patología forense, y psicología social. El International Journal of HumanComputer Studies presenta un SJR Q2 en las áreas de inteligencia artificial, psicología experimental y cognitiva, e interacción humano/computador. Finalmente, el International Journal of Information Management tiene un SJR Q2 en el área de sistema de manejo de la información.

Tabla 2. Artículos más citados en el periodo de observación.

\section{Referencia}

Zhang, L. F. (2004). Field-dependence/independence: cognitive style or perceptual ability? validating against thinking styles and academic achievement. Personality and Individual Differences 37(6), 12951311.

Akdemir, O. y Koszalka, T. A. (2008). Investigating the relationships among instructional strategies and learning styles in online environments. Computers \& Education 50(4), 1451-1461.

Chen, S. Y. y Macredie, R. (2010). Web-based interaction: A review of three important human factors. International Journal of Information Management 30(5), 379-387.

Emmett, D.; Clifford, B. R. y Gwyer, P. (2003). An investigation of the interaction between cognitive style and context reinstatement on the memory performance of eyewitnesses. Personality and Individual Differences 34(8), 1495-1508.

Frias-Martinez, E.; Chen, S. Y. y Liu, X. (2008). Investigation of behavior and perception of digital library users: A cognitive style perspective. International Journal of Information Management 28(5), 355365.

Ling, C. y Salvendy, G. (2009). Effect of evaluators' cognitive style on heuristic evaluation: Field dependent and field independent evaluators. International journal of human-computer studies 67(4), 382393.

Angeli, C.; Valanides, N. y Kirschner, P. (2009). Field dependenceindependence and instructional-design effects on learners' performance with a computer-modeling tool. Computers in Human Behavior 25(6), 1355-1366.
Número de citas a 2013

60

54
34

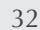

25

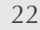

20 


\section{Referencia}

Chen, L. H. (2011). Enhancement of student learning performance using personalized diagnosis and remedial learning system. Computers \& Education 56(1), 289-299.

Chen, C. M. y Tsai, Y. N. (2012). Interactive augmented reality system for enhancing library instruction in elementary schools. Computers \& Education 59(2), 638-652.
Número de citas a 2013

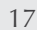

10

Las palabras clave identificadas en esta revisión comprenden 154 entradas. El término cognitive style fue usado en 42 de las 59 publicaciones señaladas (correspondiente al 71\%), seguido por la expresión field dependenceindependence usado en 11 artículos. Foreing countries y problem solving tuvieron siete usos cada una, mientras que las palabras individual differences, logical thinking y reading comprehension, fueron usadas por cinco artículos.

\section{Caracterización de autores}

Los 59 estudios consultados en esta revisión convocaron a 87 investigadores. No obstante, solamente 9 autores han publicado dos o más artículos entre 2003 y 2013. La tabla 3 muestra que Dimitrios Stamovlasis, profesor del Departamento de Filosofía y Educación de la Aristotle University of Thessaloniki (Grecia), es el autor más prolífico con 5 estudios sobre DIC publicados en revistas objetivo de este análisis.

Tabla 3. Autores con dos o más publicaciones sobre DIC en el periodo.

\begin{tabular}{|c|c|}
\hline Autor & Número de artículos \\
\hline Stamovlasis, D & 5 \\
\hline Angeli, C & 4 \\
\hline Chen, S. Y & 4 \\
\hline Tsaparlis, G. & 3 \\
\hline Valanides, N. & 3 \\
\hline Ipek, I. & 2 \\
\hline Liu, X. & 2 \\
\hline Papageorgiou, G. & 2 \\
\hline Tsitsipis, G & 2 \\
\hline
\end{tabular}

La colaboración resulta ser una de las características de la investigación en la sociedad contemporánea, en la actualidad son los equipos de investigación más que investigadores independientes los que contribuyen a un área de desarrollo. Se calculó el número de artículos con un solo autor y en colaboración (con coautoría) (tabla 4). El 38,5\% de los artículos fue escrito por un solo autor, un porcentaje idéntico (referido a 23 artículos) fue escrito con un solo coautor. 
Tabla 4. Número de autores en relación con el número de artículos publicados.

Número de autores

4
Número de publicaciones

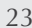

23

8

5

\section{Datos geográficos}

Se realizó un análisis de las publicaciones respecto al país en que se desarrolló el estudio. Las figuras 2 y 3 muestran los países y los continentes que contribuyeron en el desarrollo de las publicaciones examinadas. Como se nota en la figura 2, es alto el número de publicaciones que no reportan datos de lugar de desarrollo del procedimiento (13\%). Estados Unidos resulta ser el mayor generador de la investigación en DIC con 12 artículos, seguidos por Taiwán con 7 estudios e Irán con 6.

Figura 2. Países en que se han desarrollado estudios sobre DIC en el periodo de observación.
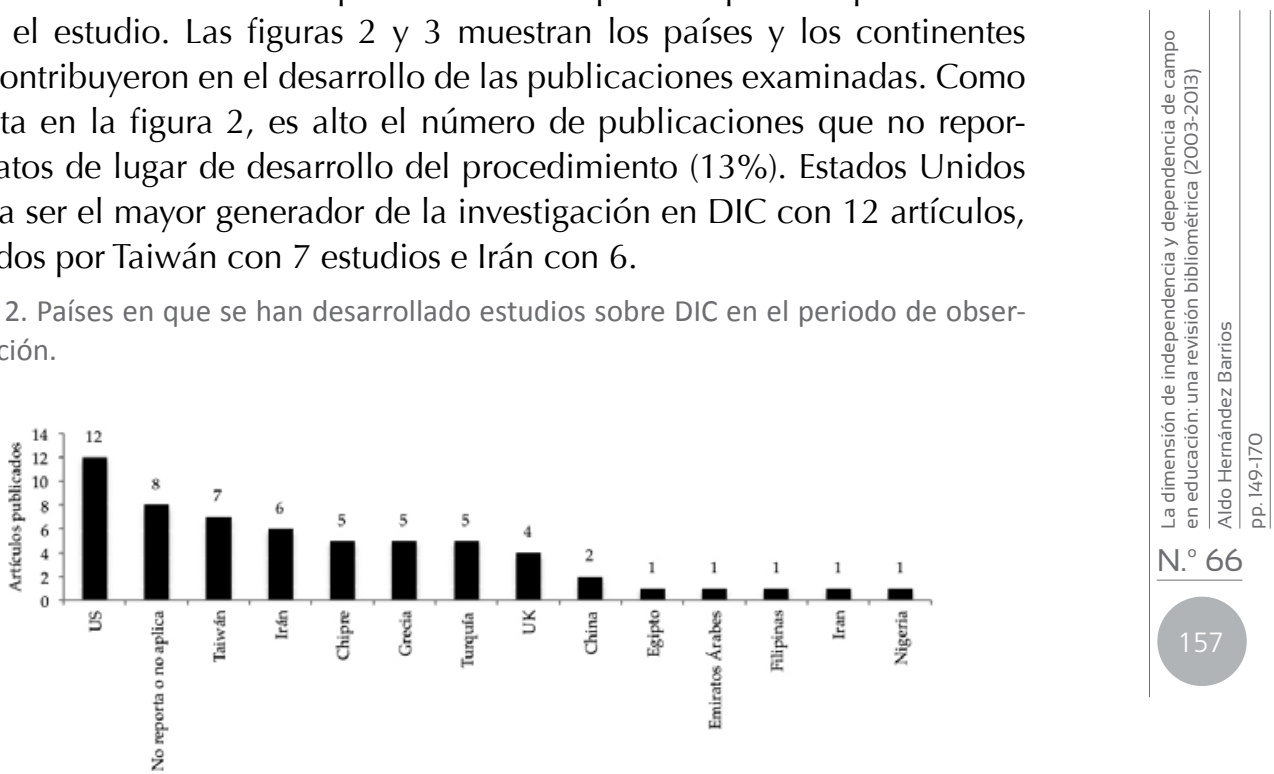

Figura 3. Números de artículos publicados en relación con el continente.

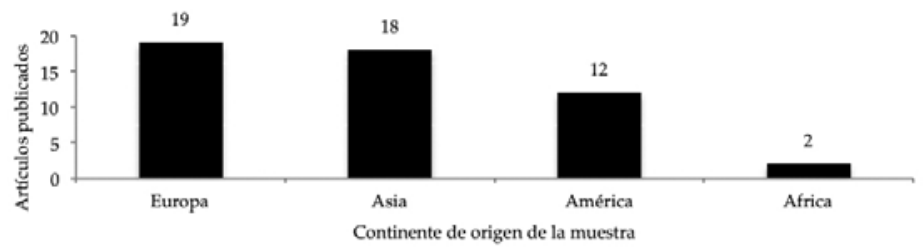

Un análisis asociado con el continente se presenta en la figura 3. Los países europeos aportan $32 \%$ de la investigación en el área de interés, seguidos por Asia con $31 \%$ y posteriormente por América con $32 \%$; no obstante, cabe indicar que ningún país adicional a Estados Unidos figura como pro- 
ductor de conocimiento sobre el área en América. El continente africano solamente ha producido dos estudios empíricos en el campo en la década de análisis.

Respecto al número de citaciones del artículo y el país en que se desarrollaron los estudios, Europa y Asia han aportado dos artículos, a cada uno con citas mayores a 40. Igualmente se pudo identificar que no hay ninguna relación entre el país o continente respecto al tipo de estudios desarrollados.

Cuarenta y siete de los 59 artículos reportaron el contexto en el que se desarrollaron los estudios, 7 artículos no contenían dicha información (sin considerar los 5 estudios de carácter documental). En la figura 4 se presenta un histograma en que se relaciona el contexto del estudio y el número de artículos. De acuerdo con la figura 4, 32 artículos fueron desarrollados con participantes de contextos universitarios, 10 con participantes de educación básica, 4 de educación secundaria y un estudio reportó participantes de instituciones tecnológicas.

Figura 4. Contexto en el que se realizaron las investigaciones.

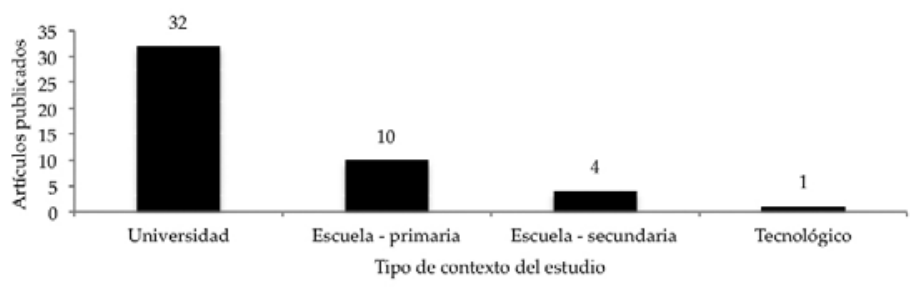

\section{Tipos de estudios y equipos de investigación}

En cuanto a los tipos de estudios identificados, los estudios correlacionales constituyen la mayor frecuencia observada; de los 59 artículos, 34 son correlacionales (58\%). Los estudios experimentales (11 artículos) ocupan el segundo puesto entre las metodologías usadas para indagar sobre dependencia e independencia de campo. En relación con los estudios documentales (con 5 artículos), 2 de ellos son teóricos y el resto son revisiones parciales (figura 5). En la ventana de observación no se encuentra ningún tipo de relación entre el año y el tipo de estudio desarrollado.

La tabla 5 muestra la relación entre el número de autores participantes en la elaboración de los artículos. De manera particular, los estudios correlaciónales (tipo más frecuente identificado en esta revisión) tiene mayor frecuencia de colaboración. 
Figura 5. Frecuencia de tipos de estudios realizados desde 2003 a 2013.

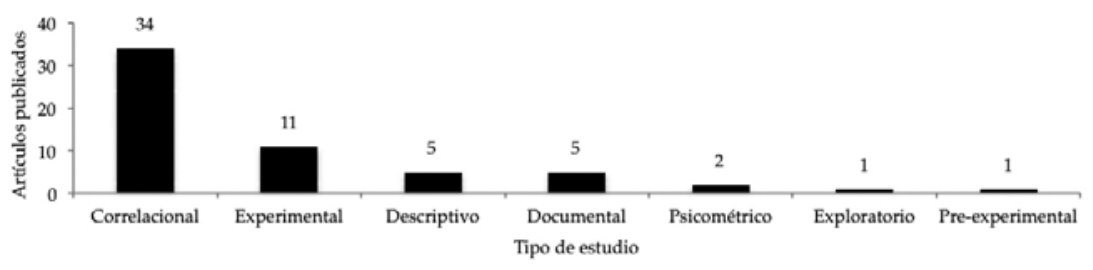

Tabla 5. Tipo de estudio y colaboración.

\begin{tabular}{|c|c|c|c|c|}
\hline & \multicolumn{4}{|c|}{ Número de autores } \\
\hline Tipo de estudio & $\mathbf{1}$ & $\mathbf{2}$ & $\mathbf{3}$ & $\mathbf{4}$ \\
\hline Correlacional & 11 & 14 & 5 & 4 \\
\hline Descriptivo & 3 & 2 & 0 & 0 \\
\hline Documental & 2 & 2 & 1 & 0 \\
\hline Experimental & 4 & 4 & 2 & 1 \\
\hline Exploratorio & 1 & 0 & 0 & 0 \\
\hline Preexperimental & 1 & 0 & 0 & 0 \\
\hline Psicométrico & 1 & 1 & 0 & 0 \\
\hline
\end{tabular}

\section{Variables e instrumentos}

Se analizaron las variables estudiadas en los artículos consultados. Sesenta y cuatro variables fueron identificadas. La de mayor frecuencia de uso fue independencia-dependencia de campo con 46 usos, seguida por solución de problemas (5 usos), pensamiento lógico (4 usos), instrucción basada en computadores (3 usos) y estilo cognitivo, memoria de trabajo y pensamiento convergente-divergente (con 2 usos cada una) (tabla 6).

Tabla 6. Uso de las variables en los estudios sobre DIC.

\begin{tabular}{|l|c|}
\hline \multicolumn{1}{|c|}{ Variable } & Número de artículos \\
\hline Independencia-dependencia de Campo & 46 \\
\hline Solución de problemas & 5 \\
\hline Pensamiento lógico & 4 \\
\hline Instrucción basada en computadores & 3 \\
\hline Estilo cognitivo & 2 \\
\hline Memoria de trabajo & 2 \\
\hline Pensamiento convergente-divergente & 2 \\
\hline Solución de problemas & 2 \\
\hline
\end{tabular}


La instrumentación usada en los estudios consultados es muy variada, un total de 39 instrumentos de evaluación diferentes fueron identificados. De acuerdo con la tabla 7, el Group Embedded Figure Test (GEFT) es el más utilizado; el 54\% de los estudios lo emplearon para evaluar la independencia de campo. Otros instrumentos empleados más de una vez son listados en la tabla 7.

Tabla 7. Instrumentos usados en los estudios.

\begin{tabular}{|c|c|}
\hline Instrumento & Número de usos \\
\hline Group Embedded Figure Test (GEFT) & 32 \\
\hline $\begin{array}{l}\text { Pruebas de desempeño académico (desarrolladas por los } \\
\text { investigadores) }\end{array}$ & 9 \\
\hline Hidden Figure Test (HFT) & 5 \\
\hline $\begin{array}{l}\text { Prueba de razonamiento formal de Lawson (versión papel- } \\
\text { lápiz) }\end{array}$ & 5 \\
\hline Cognitive Style Analyisis (CSA) & 3 \\
\hline Digit Span Backwards Test - subescala del WAIS & 4 \\
\hline
\end{tabular}

\section{Discusión}

El propósito de este estudio fue hacer una revisión de los artículos publicados en las revistas científicas sobre independencia y dependencia de campo en el área de la educación entre los años 2003 y 2013 (incluidos los artículos aceptados para publicación).

A partir de la cobertura de revistas que tienen las bases de datos y los criterios de exclusión de la información, aplicados posteriormente a la búsqueda experta, no es posible pensar que esta revisión cubra la totalidad de las publicaciones en el área de interés. No obstante, y como se justificó la elección de las bases de datos, puede considerarse una muestra representativa de la producción académica e investigativa en idioma inglés más influyente en el mundo de la ciencia, asociada con los estilos cognitivos en su dimensión dependencia e independencia de campo.

El estudio de la DIC en el área de la educación, se evidencia una producción total de 59 artículos en un periodo de 10 años, aporta en promedio 10 artículos por año a la discusión sobre estilos cognitivos para la psicología educativa y las áreas asociadas a la educación en general. Sin embargo, de acuerdo con la figura 1, el rango de variación está entre 2 y 11 artículos, con picos evidentes (años 2004, 2010 y 2012) aspecto que 
puede ser interpretado como que el programa de investigación en DIC presenta interrupciones. Si bien es claro que el índice de inmediatez (tiempo que transcurre entre el sometimiento editorial de un artículo y su publicación) oscila de 6 meses a un año y medio, un programa de investigación tendería a mostrar una tasa constante de publicaciones, así como un área nueva de interés presentaría una tendencia de crecimiento positiva. Se plantean dos hipótesis, la primera consiste en que la variabilidad en la cantidad de publicaciones sobre DIC puede deberse a que no resulta ser el tema principal de interés para los investigadores, y la segunda, que los artículos en el área presentan algunas dificultades para su catalogación y en consecuencia la disminución de las probabilidades de publicación en bases de datos referentes.

Una hipótesis como la primera requiere un examen mucho más profundo. De acuerdo con la información suministrada en la tabla 3, 9 autores (de 87) han participado en la discusión académica/investigativa en este periodo con 2 o más publicaciones; el autor más prolífico ha realizado 5 publicaciones (a razón de un artículo cada dos años). Esta información, unida a la característica de colaboración (tabla 4) resulta interesante; hay una dispersión de autores y un trabajo más individualizado o en duplas. Sería conveniente reconocer que los programas de investigación se fortale- cen a partir del trabajo colaborativo de los investigadores con trayectoria con los estudiantes doctorales y los pasantes de posdoctorado, aspecto que redunda en publicaciones con mayores indicadores de colaboración. En áreas básicas y aplicadas/ profesionales resulta evidente el trabajo de equipos de investigación (por ejemplo, bioquímica, medicina y psicología, entre otras).

Las palabras clave representan una característica de catalogación típica de las publicaciones científicas. Los hallazgos de esta revisión señalan una alta dispersión en cuanto a palabras clave; 6 palabras clave presentan un uso relativamente frecuente, lo que implica que 149 palabras clave tiene un uso por una o máximo dos veces. Tal dispersión tiene por lo menos dos interpretaciones, la primera es que las palabras clave se plantean a expensas de ignorar los Thesaurus, y la segunda, es que una búsqueda centrada en los desempeños (particularmente en el área de la educación) para la configuración de referentes teóricos y empíricos puede dejar de lado información relevante. Vale la pena identificar que algunas de las palabras clave podrían categorizarse en torno a los aspectos asociados con uso de TIC o ambientes virtuales de aprendizaje, hecho que tal vez refleje la orientación dada por las agencias financiadoras de investigaciones. Resulta evidente que la DIC está siendo estudiada en relación a los virtuales de aprendizaje (e-lear- 
ning y bi-learning), pero la falta de unicidad en los criterios para establecer palabras clave redunda en que haya descriptores tangenciales de la temática.

Resulta evidente considerar que estilos cognitivos e independencia y dependencia de campo sean las variables con mayor frecuencia de uso, dado que constituyen los elementos críticos de la búsqueda sistemática y fungen como variables independientes o predictoras. Resulta interesante que en la muestra de artículos 54 variables (fueran predictoras o criterio) tuvieron una frecuencia igual a 1. Este hallazgo respalda la dispersión de los esfuerzos investigativos en torno a la DIC.

Las características sociodemográficas de las muestras estudiadas (figura 4) indican que los estudiantes universitarios resultan ser los individuos preferidos para los estudios. Por lo general, los estudiantes universitarios son población cautiva para las investigaciones y a la vez resultan ser sobreevaluados. El tipo de participante y el tipo de estudio (prevalentes los de orden correlacional) son factores que mejoran la factibilidad y viabilidad de los estudios, pero a expensas de no caracterizar el fenómeno a través del tiempo (por ejemplo, a través de estudios longitudinales). En consecuencia se pierde la posibilidad de identificar aspectos del entorno y experiencias críticas que den cuenta de posibles cambios en la dimensión DIC.

Respecto a la segunda hipótesis planteada, asociada a dificultades de catalogación de los artículos, algunos elementos han sido señalados con anterioridad; la diversidad de palabras clave (por ejemplo, las variables y las palabras clave). No obstante, el aspecto más crítico identificado en esta revisión consiste en la carencia de información sociodemográfica de los participantes de los estudios; datos como edad, sexo, procedencia, tipo de institución son necesarios e indicados por el estilo editorial APA (2009). Esta resulta ser una seria limitante a la hora de realizar estudios metaanalíticos, al igual de la carencia de datos asociados con los cálculos de potencia de la muestra y tamaños del efecto; los cuales no se presentaron en ninguno de los estudios revisados. Una posible razón de esta carencia de cálculos reside en los bajos tamaños muestrales y, sobre todo, en el tipo de diseño muestral implicado; ninguno de los estudios refirió alguna estrategia paramétrica para diseñar la muestra.

Por otra parte, y tomando distancia de las hipótesis planteadas al inicio de la discusión, esta revisión favorece la identifi- 
cación de un conjunto de revistas prototípicas de referencia. El total de artículos de la revisión se distribuyen en 42 revistas diferentes, lo que indica en términos matemáticos que una distribución homogénea implicaría un artículo publicado en cada una de ellas. Diez revistas han publicado el $38 \%$ de los artículos en el área. Vale la pena resaltar que las dos revistas con mayor frecuencia de publicación en DIC tiene una política editorial orientada a los aspectos tecnológicos (Computers \& Education y Educational Tecnology Research and Development).

Respecto a la ubicación geográfica de los integrantes de las muestras, Estados Unidos es el que contribuye con más muestras a la investigación. Otros países, particularmente europeos y asiáticos aportan al conocimiento en el área. Se muestra un esfuerzo de los países africanos por entrar en el escenario de discusión académica en el área; es evidente la falta de países de Latinoamérica y el Caribe. La barrera idiomática puede ser un factor clave para el rezago de países no industrializados en el escenario académico de discusión. Prueba de lo anterior es soportada por el trabajo de Hederich (2013), quien reporta 9 artículos sobre DIC entre 1998 y 2011 a partir del análisis de la base SciElo orientada a Iberoamérica.

En los artículos identificados en la búsqueda, que muestran revisiones parciales del área, no se identifican esfuerzos teóricos basados en la evidencia para hacer avances en la conceptualización. Se han realizado esfuerzos integradores en el aspecto conceptual en el área (por ejemplo, Curry, 1987; Messik, 1986; Riding y Cheema, 1991), pero no son evidentes en más de tres artículos de la revisión. No obstante, la dimensión de dependencia e independencia de campo es un constructo psicológico por naturaleza. Una búsqueda en el Diccionario Conciso de Psicología (American Psychological Association, 2010) permite ubicar el término estilo cognitivo y en su definición se señala explícitamente la DIC; igualmente se pueden ubicar las entradas independencia de campo y dependencia de campo. El hecho de que un diccionario rector disciplinar contenga estas entradas señala su posicionamiento y uso en la comunidad científica.

El fenómeno psicológico implicado en el constructo estilo cognitivo en su dimensión de independencia y dependencia de campo resulta relevante, ya que ha resultado ser predictor de muchas ejecuciones en diferentes ámbitos de la conducta humana, el 95\% de los artículos empíricos revisados indican mayor efectividad de los individuos independientes de campo en diferentes tareas de orden académico. Un examen juicioso de la conceptualización del fenómeno parece ser necesario para poder avanzar en investigación básica y sobre todo en investigación aplicada (particularmente en el área educativa); revisitar el 
constructo y sus categorías conceptuales asociadas permitirá, por una lado, la reducción de la dispersión de intereses investigativos en el campo educativo, por otro, el desarrollo o sofisticación de instrumentos de evaluación y de estudios longitudinales que den cuenta del fenómeno.

\section{Referencias bibliográficas}

American Psychological Association. (2009). Publication Manual of the American Psychological Association. 6a. ed. Washington, DC: American Psychological Association.: American Psychological Association.

American Psychological Association. (2010). APA Diccionario Conciso de Psicología. México: El Manual Moderno.

Chao, L.; Huang, J. y Li, A. (2003). A study of field independence versus field dependence of school teachers and university students in mathematics. Perceptual and Motor Skills 97(3), 873-876.

Curry, L. (1987). Integrating concepts of cognitive or learning style: A review with attention to psychometric standards. Ottawa, ON: Canadian College of Health Service Executives.

Flores, T. D. (2012). DSM-IV Manual diagnóstico y estadístico de los trastornos mentales. Barcelona: Masson.

Hederich, C. (2010). Acerca de la noción general de estilo en la educación pertinencia, importancia y especificidad. Actualidades Pedagógicas, 55, 13-21.

Hederich, C. (2013). Estilística educativa. Revista Colombiana de Educación, 64, 21-56.

Hederich, C. y Camargo, A. (2000). Estilo cognitivo y logro académico en la ciudad de Bogotá. Revista Colombiana de Educación, 40-41, 147-172.

Hudson, T.E.; Li, W. y Matin, L. (2006). The field dependence/ independence cognitive style does not control the spatial perception of elevation. Perception \& Psychophysics 68(3), 377-392.

López, O.; Hederich, C. y Camargo, A. (2011). Estilo cognitivo y logro académico. Educación y Educadores 14(1), 67-82.

Messick, S (1984) The nature of cognitive styles: Problems and promise in educational practice. Educational Psychologist, 19, 59-74.

Petticrew, M. y Roberts, H. (2008). Systematic reviews in the social sciences: A practical guide. Nueva York: Wiley. 
Riding, R. J. y Cheema, I. (1991). Cognitive Styles-An Overview and Integration. Educational Psychology 11(4), 193-215.

Saracho, O. (1980). The Relationship between the Teachers' Cognitive Style and Their Perceptions of Their Students' Academic Achievements. Educational Research Quarterly 5(3), 40-49.

Tinajero, C. y Fernández, M. F. P. (1998). Field dependenceindependence in second-languge acquisition: Some forgotten aspects. Spanish Journal of Psychology 1(1), 32-38.

Tinajero, C.; Castelo, A.; Guisande, A. y Páramo, F. (2011). Adaptive teaching and field dependence-independence: Instructional implications. Revista Latinoamericana de Psicología 43(3), 497-510.

Witkin, H. (1950). Individual differences in the case of perception of embedded figures. Journal of Personality, 19, 1-16.

Witkin, H. (1964). Origins of Cognitive Style En: C. Scheere (ed.) Cognition: Theory, Research, Promise (pp. 172-205). Nueva York: Harper.

Witkin, H. (1965). Psychological differentiation and forms of pathology. Journal of Abnormal Psychology, 70, 317-336.

Witkin, H. (1976). Cognitive style in academic performance and in teacher student relations En: S. Messick et al. (eds). Individuality in Learning. San Francisco: Jossey-Bass.
Witkin, H. y Asch, S.E. (1948). Studies in Space Orientation. III Perception of the Upright in the Absence of a Visual Field. Journal of Experimental Psychology, 38, 603-614.

\section{Artículos examinados para la revisión}

Adegoke, B. A. (2011). Effect of indirect teacher influence on dependent-prone students' learning outcomes in secondary school mathematics. Electronic Journal of Research in Educational Psychology 9(1), 283-308.

Akdemir, O. y Koszalka, T. A. (2008). Investigating the relationships among instructional strategies and learning styles in online environments. Computers \& Education 50(4), 14511461.

Alamolhodaei, H. (2009). A working memory model applied to mathematical word problem solving. Asia Pacific Education Review 10(2), 183-192.

Almekhlafi, A. G. (2006). Effectiveness of Interactive Multimedia Environment on Language Acquisition Skills of 6th Grade Students in the United Arab Emirates. International Journal of Instructional Media 33(4), 427-441.

Altun, A. y Cakan, M. (2006). Undergraduate students' academic achievement, field dependent/ independent cognitive styles 
and attitude toward computers. Journal of Educational Technologyand Society 9(1), 289.

Angeli, C. (2013). Examining the Effects of Field DependenceIndependence on Learners' Problem-Solving Performance and Interaction with a Computer Modeling Tool: Implications for the Design of Joint Cognitive Systems. Computers \& Education. 62, 221-230.

Angeli, C. y Valanides, N. (2004). Examining the effects of textonly and text-and-visual instructional materials on the achievement of field-dependent and field-independent learners during problem-solving with modeling software. Educational Technology Research and Development 52(4), 23-36.

Angeli, C. y Valanides, N. (2004). Examining the effects of textonly and text-and-visual instructional materials on the achievement of field-dependent and field-independent learners during problem-solving with modeling software. Educational Technology Research and Development 52(4), 23-36

Angeli, C. y Valanides, N. (2013). Using educational data mining methods to assess field-dependent and field-independent learners' complex problem solving. Educational Technology Research and Development, 1-28.

Angeli, C.; Valanides, N. y Kirschner, P. (2009). Field dependence-independence and instructional-design effects on learners' performance with a computer-modeling tool. Computers in Human Behavior 25(6), 1355-1366.

Aragon, S. R. (2004). Information processing patterns of postsecondary American Indian/Alaska native students. Journal of American Indian Education 43(3), 1-20.

Ates, S. y Cataloglu, E. (2007). The effects of students' cognitive styles on conceptual understandings and problem-solving skills in introductory mechanics. Research in Science \& Technological Education 25(2), 167-178.

Baker, R. M. y Dwyer, F. (2005). Effect of Instructional Strategies and Individual Differences: A Meta-Analytic Assessment. International journal of instructional media 32(1), 69.

Chapelle, C. A. y Heift, T. (2009). Individual learner differences in CALL: The field independence/dependence (FID) construct. Calico Journal 26(2), 246-266. 
Chen, C. J. y Liu, P. L. (2012). Comparisons of Learner-Generated versus Instructor-Provided Multimedia Annotations. Turkish Online Journal of Educational Technology-TOJET, 11(4), 72-83.

Chen, C. M. y Tsai, Y. N. (2012). Interactive augmented reality system for enhancing library instruction in elementary schools. Computers \& Education 59(2), 638-652.

Chen, L. H. (2011). Enhancement of student learning performance using personalized diagnosis and remedial learning system. Computers \& Education 56(1), 289-299.

Chen, S. Y. y Liu, X. (2011). Mining students' learning patterns and performance in Web-based instruction: a cognitive style approach. Interactive Learning Environments 19(2), 179-192.

Chen, S. Y. y Macredie, R. (2010). Web-based interaction: A review of three important human factors. International Journal of Information Management 30(5), 379-387.

Clewley, N.; Chen, S. Y. y Liu, X. (2011). Mining Learning Preferences in Web-based Instruction: Holists vs. Serialists. Educational Technology \& Society, 14(4), 266-277.

Damusis, V. y Desjarlais, L. (2004). La dépendance-independance du champ comme style cognitif: certaines consequences psychopedagogiques. Revue Européenne de Psychologie Appliquée/European Review of Applied Psychology 54(2), 65-72.

Duman, B. y Celik, O. (2012). Cognitive Scenario Writing and Concept-Using Skills According to Cognitive Styles. Hacettepe University Journal of Education, 42, 163-174.

Emmett, D.; Clifford, B. R. y Gwyer, P. (2003). An investigation of the interaction between cognitive style and context reinstatement on the memory performance of eyewitnesses. Personality and Individual Differences 34(8), 1495-1508.

Frias-Martinez, E.; Chen, S. Y. y Liu, X. (2008). Investigation of behavior and perception of digital library users: A cognitive style perspective. International Journal of Information Management, 28(5), 355-365.

Ghabanchi, Z. (2010). The Effectiveness of Incidental Teaching of Grammar to Iranian Students. Journal of College Teaching \& Learning 7(1), 71-78.

Handal, B. y Herrington, A. (2004). On being dependent or independent in computer based learning environments. E-Journal of Instructional Science and Technology 7(2).

Hite, C. E. (2004). Expository content area texts, cognitive style and gender: Their effects on reading comprehension. Literacy Research and Instruction 43(4), 41-74. 
Holmes, R. M.; Liden, S. y Shin, L. (2013). Children's Thinking Styles, Play, and Academic Performance. American Journal of Play 5(2), 219-238.

Hong, J. C.; Hwang, M. Y.; Tam, K. P.; Lai, Y. H. y Liu, L. C. (2012). Effects of cognitive style on digital jigsaw puzzle performance: A GridWare analysis. Computers in Human Behavior 28(3), 920-928.

Hsieh, S. W. (2011). Effects of Cognitive Styles on an MSN Virtual Learning Companion System as an Adjunct to Classroom Instructions. Educational Technology \& Society 14(2), 161-174.

Ipek, I. (2010). The Effects of CBI Lesson Sequence Type and Field Dependence on Learning from Computer-Based Cooperative Instruction in Web. Turkish Online Journal of Educational Technology-TOJET 9(1), 221-234.

Ipek, I. (2011). The Effects of Text Density Levels and the Cognitive Style of Field Dependence on Learning from a CBI Tutorial. Turkish Online Journal of Educational Technology-TOJET 10(1), 167-182.

Klein, A. M. (2008). Sensitivity to the Learning Needs of Newcomers in Foreign Language Settings. Multicultural Education 16(2), 41-44.

Ling, C. y Salvendy, G. (2009). Effect of evaluators' cognitive style on heuristic evaluation: Field dependent and field independent evaluators. International journal of human-computer studies 67(4), 382-393.

Liu, W. y Chepyator-Thomson, J. R. (2009). Field dependenceindependence and physical activity engagement among middle school students. Physical Education and Sport Pedagogy 14(2), 125-136.

Moosavi, S.; Radmehr, F. y Hlamolhodaei, H. (2012). The role of mathematical homework and prior knowledge on the relationship between students' mathematical performance, cognitive style and working memory capacity. Electronic Journal of Research in Educational Psychology, 10(3), 1223-1248.

Naimie, Z.; Abuzaid, R. A.; Siraj, S.; Shagholi, R. y Hejaili, H. A. (2010). Do you know where I can find the new center which is called "Cognitive styles and language learning strategies link"?. Procedia-Social and Behavioral Sciences 2(2), 497-500. 
Nicolaou, A. A. y Xistouri, X. (2011). Field dependence/independence cognitive style and problem posing: an investigation with sixth grade students. Educational Psychology 31(5), 611627.

Rezaee, M. y Farahian, M. (2012). The Case Study of a FieldIndependent English Language Learner. Procedia-Social and Behavioral Sciences, 47, 114119.

Rittschof, K. A. (2010). Field dependence-independence as visuospatial and executive functioning in working memory: Implications for instructional systems design and research. Educational Technology Research and Development 58(1), 99-114.

Salmani-Nodoushan, M. A. (2007). Is Field Dependence or Independence a Predictor of EFL Reading Performance?. TESL Canada Journal 24(2), 82-108.

Saracho, O. (2003). Matching teachers' and students' cognitive styles. Early Child Development and Care 173(2-3), 161-173.

Soureshjani, K. H. y Safikhani, A. (2012). Reconsidering metaphorical performance of efl learners with a focus on their cognitive features. Journal of Pan-Pacific Association of Applied Linguistics 16(1), 19-32.

St Clair-Thompson, H.; Overton, T. y Botton, C. (2010). Information processing: a review of implications of Johnstone's model for science education. Research in Science \& Technological Education 28(2), 131-148.

Stamovlasis, D. y Papageorgiou, G. (2012). Understanding chemical change in primary education: The effect of two cognitive variables. Journal of Science Teacher Education 23(2), 177-197.

Stamovlasis, D. y Tsaparlis, G. (2005). Cognitive variables in problem solving: A nonlinear approach. International Journal of Science and Mathematics Education 3(1), 7-32.

Stamovlasis, D. y Tsaparlis, G. (2012). Applying catastrophe theory to an information-processing model of problem solving in science education. Science Education 96(3), 392-410.

Tomes, Y. I. (2008). Ethnicity, Cognitive Styles, and Math Achievement: Variability within AfricanAmerican Post-Secondary Students. Multicultural Perspectives 10(1), 17-23.

Torres, R. M. y Ulmer, J. D. (2007). An investigation of time distribution of pre-service teachers while interning. Journal of Agricultural Education 48(2), 1-12.

Tsaparlis, G. (2005). Non-algorithmic quantitative problem solving in university physical chemistry: a correlation study of the role of selective cognitive factors. Research in Science \& Technological Education 23(2), 125-148.

Tsitsipis, G.; Stamovlasis, D. y Papageorgiou, G. (2010). The effect 
of three cognitive variables on students' understanding of the particulate nature of matter and its changes of state. International Journal of Science Education 32(8), 987-1016.

Tsitsipis, G.; Stamovlasis, D. y Papageorgiou, G. (2012). A probabilistic model for students'errors and misconceptions on the structure of matter in relation to three cognitive variables. International Journal of Science and Mathematics Education 10(4), 777-802.

Vincent-Morin, M. y Lafont, L. (2005). Learning-Method Choices and Personal Characteristics in Solving a Physical Education Problem. Journal of Teaching in Physical Education 24(3), 226-242.

Wang, T. H. (2007). What strategies are effective for formative assessment in an e-learning environment? Journal of Computer Assisted Learning 23(3), 171-186.

Wilson, S. B. (2005). Computer assisted fluid power instruction: A comparison of hands-on and computer-simulated laboratory experiences for post-secondary students. Journal of Technology Studies 31(1), 57-61.

Yan, J. H. (2010). Cognitive styles affect choice response time and accuracy. Personality and Individual Differences 48(6), 747-751.

Zhang, L. F. (2004). Field-dependence/independence: cognitive style or perceptual ability? - validating against thinking styles and academic achievement. Personality and Individual Differences 37(6), 1295-1311.

Zheng, R. (2010). Effects of Situated Learning on Students' Knowledge Acquisition: An Individual Differences Perspective. Journal of Educational Computing Research 43(4), 467487.

Zheng, R. Z.; Yang, W.; Garcia, D. y McCadden, E. P. (2008). Effects of multimedia and schema induced analogical reasoning on science learning. Journal of computer assisted learning 24(6), 474-482. 